\title{
Surface viscometry in a uniform magnetic field
}

\author{
J. Delacroix ${ }^{1, a}$ And L. Davoust ${ }^{2}$ \\ 1 University of Grenoble Alpes, Materials \& Processes Science \& Engineering (SIMaP) Laboratory, Electromagnetic Processing \\ of Materials (EPM) Group, 38402 St-Martin d'Hères, France \\ 2 Grenoble Institute of Technology (Grenoble-INP), CNRS, Materials \& Processes Science \& Engineering (SIMaP) Laboratory, \\ Electromagnetic Processing of Materials (EPM) Group, 38402 St-Martin d'Hères, France
}

Received 2 December 2015, Accepted 24 April 2016

\begin{abstract}
This paper addresses an original numerical coupling between surface mechanics of a gradually oxidizing liquid metal surface, and a supporting annular MHD flow, in the general layout of the classical annular viscometer, originally developed by Mannheimer et al. [J. Colloid Interface Sci. 32 (1970) 195-211]. A purely hydrodynamic interplay between a main azimuthal flow (induced by a rotating floor) and a secondary overturning flow generated by centrifugation is found to be strongly affected by both surface viscous shear and surface viscous dilatation. When centrifugation competes with electromagnetic effects, advection of the main flow by the secondary flow is proved to affect significantly the core MHD flow, leading to original MHD flow patterns. The latter phenomenology reveals to be relevant to characterise the surface viscosities of a gradually oxidising liquid metal surface.
\end{abstract}

Key words: MHD / annular flow / surface viscosities / Hartmann layers

\section{Introduction}

Modeling of magnetohydrodynamic (MHD) two-phase flows has recently become an issue of major interest, given the numerous industrial applications potentially affected. It potentially affects many fields, such as metallurgy (stirring by bubble plumes in reactors [1]), microelectronics (MHD-driven metal cooling processes [2]), or nuclear fusion technology (two-phase MHD issues with the breeder blanket based cooling loop [3]).

To our knowledge, little is actually known about the surface rheology of MHD flows, e.g. when a liquid metal is progressively contaminated through oxidation processes. On the one hand, the viscoelastic properties of liquid metals have been experimentally investigated by researchers including Kolevzon et al. [4,5], Larsen et al. [6] or Liu et al. [2], who each highlighted radically different mechanical behavior characteristics that depend on the level of oxidation. However, their results were not coupled with MHD.

On the other hand, the MHD of single-phase laminar flows has been extensively studied for many years. The flow can be either confined (see Shercliff [7]), or may have a free surface - for a Couette flow with a free surface, see e.g. Lehnert [8]. However, the fundamental issue of varying boundary conditions would seem to have only been considered from an electrical point of view. Thus, in the case of duct flows, the walls can have infinite

\footnotetext{
${ }^{a}$ Corresponding author: jules.delacroix@cea.fr
}

electrical conductivity (see Shercliff [7]), no conductivity (Moreau [9]), mixed infinite and vanishing conductivities (Hunt et al. [10]), or arbitrary conductivity (Tabeling et al. [11]). The electrical influence of the walls, governed by the ratio of bulk and wall electrical conductivities $\sigma / \sigma_{\mathrm{w}}$, completely modifies the electric circuit, and results in a major impact on the topology of the MHD flow.

In this study, the same kind of general approach for the boundary conditions is taken, but this time from a mechanical point of view. In this respect, for flows including liquid/gas interfaces, the competition between bulk MHD and surface rheology (driven by the contamination rate of the liquid surface) may lead to a mechanically varying boundary condition, suspected to affect considerably the overall MHD flow. To deal with the practical conditions of molten metals in metallurgy industry, we need to investigate surface mechanics separately from bulk flow, especially when the free surface is gradually oxidizing. The mechanical coupling can therefore be considered between a liquid surface and the underlying bulk, through the emergence of dilatational and shear surface viscosities.

\section{Outlines}

The system under consideration is an annular MHD viscometer (Fig. 1). The problem is considered 2-D axisymmetric $(\partial / \partial \theta=0)$, so that the domain can be reduced to the cross-section of an annular open channel. 


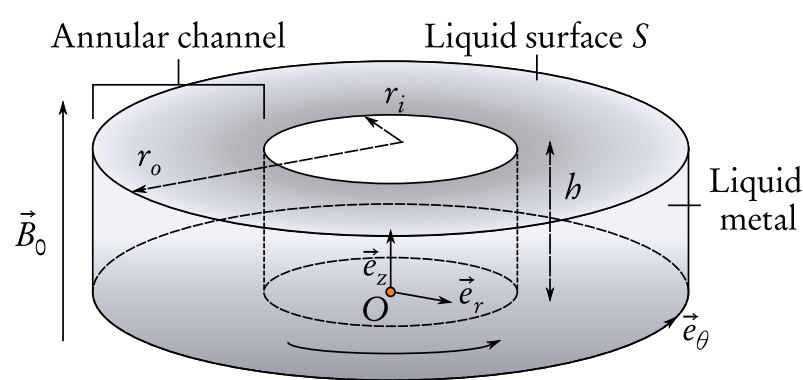

Rotating floor at angular speed $\Omega$

Fig. 1. The annular MHD viscometer.

The latter consists of a rectangular cross-section, where the inner and outer radii are, respectively, $r_{i}$ and $r_{o}$, and the height is $h$, where $h<r_{i}, r_{o}$ (indicative values: $r_{i}=3 \mathrm{~cm}, r_{o}=7 \mathrm{~cm}$ and $\left.h=1 \mathrm{~cm}\right)$. The coordinate system used is the cylindrical system $\left\{O, \vec{e}_{r}, \vec{e}_{\theta}, \vec{e}_{z}\right\}$. The annular floor of the channel rotates around the $z$ axis with a varying angular speed of $\Omega$, while the two side walls are fixed. An outer vertical permanent magnetic field $\vec{B}_{0}=B_{0} \vec{e}_{z}$ is imposed, and the channel is filled with an electroconductive fluid, e.g. a liquid eutectic alloy called Galinstan.

There are numerous interest in this configuration, which is inspired by the deep channel viscometer [12-15]. First, the electroconductive rotating fluid subjected to an outer magnetic field constitutes a MHD liquid/gas stratified flow, which is a basic study case in view of the description of different MHD two-phase flow regimes. Besides, this particular layout allows for a wide variety of physical phenomena to be enhanced, beginning with bulk effects. Thus, inertial effects can be easily highlighted by changing the value of the angular speed $\Omega$ of the rotating floor [16]. On the contrary, if the goal is to extinguish centrifugation, tuning the value of the outer magnetic field $B_{0}$ may lead to a fully 2-D MHD flow, due to the well-known two-dimensionality tendency of magnetic induction [17].

Concerning surface effects, and compared to the classical Taylor-Couette layout, the vertical shear is in this case particularly emphasized in the annular viscometer configuration, through a shallow configuration $\left(h<r_{i}, r_{o}\right)$. This means that strong velocity gradients are generated along the $\vec{e}_{z}$-axis, whereas these gradients preferentially develop along the $\vec{e}_{r}$-axis in the Taylor-Couette case. Anticipating what follows, it is shown later in Equations (12) and (13) that the coupling term between surface and sub-phase flows involves $\partial v_{r} / \partial z$ and $\partial v_{\theta} / \partial z$ terms, where $v_{r}$ and $v_{\theta}$ are the radial component and the azimuthal component of velocity, respectively. Consequently, the resulting shearing is expected to be magnified, and the impact of varying boundary conditions at the liquid surface on the overall MHD flow may be highlighted.

In the light of all these considerations, the physical insight provided by the annular MHD viscometer is expected to be significant. The goal of this $3-\mathrm{D}$ numerical study is to extend significantly the scope of a compan- ion paper [17], focused on the 2-D analytical study of the annular MHD viscometer. This benchmarking analytical study is based on the assumptions $\mathrm{Ha} \gg 1$ and $\mathrm{Ha} \gg \mathrm{Re}$, where Ha and Re are the Hartmann and Reynolds numbers, respectively. These assumptions allowed the authors to ignore the inertial effects, and to highlight solely the competition between surface viscous shearing and the effects due to Lorentz force. Typically, it has been shown that surface rheology actually monitors the electrical activation of Hartmann layers.

For the present study, no particular assumptions have been made concerning the values of Re and Ha (except that $\operatorname{Re} \leq 10^{4}$ in order to avoid turbulence issues). Consequently, a swirling flow occurs when $\mathrm{Ha}<\mathrm{Re}$, leading to inertial centrifugal effects. As a consequence, a new rheological parameter is solicited at the interface, linked to surface viscous dilatation, which might affect core flow. Furthermore, interacting with the outer magnetic field, the centrifugal effects bring an original MHD flow into play, which in turn competes with surface rheology. This peculiar strong coupling is phenomenologically investigated in this paper. This allows us to highlight a variety of atypical MHD flow patterns, as well as to determine distinct working conditions for a supporting experimental test-bench. The related "scaling laws" shall reveal most valuable for the interpretation of upcoming experimental results.

\section{Physical modelling}

\subsection{Notations and assumptions}

With respect to purely hydrodynamic assumptions, the annular shear flow considered is assumed to be a permanent, axisymmetric $(\partial / \partial \theta=0)$, incompressible and viscous Newtonian flow with no temperature dependence, so that the bulk physical properties of Galinstan are considered to be constant. The flow is laminar with $\mathrm{Re}=$ $\rho \Omega r_{o}^{2} / \eta$, where $\eta$ is the dynamic viscosity and $\rho$ is the density of Galinstan (indicative values: $\eta=2.4 \times 10^{-3}$ Pa.s and $\rho=6.4 \times 10^{-3} \mathrm{~kg} \cdot \mathrm{m}^{-3}$, see Liu et al. [2]). In this paper, and unlike in the supporting analytical study [17] where Re is set to be sufficiently small, Re varies in order to study inertial effects, and increased up to the onset value $R e \leq 10^{4}$, in order to avoid turbulence issues (see Serre et al. [18] for similar turbulence considerations).

Moreover, the quasi-static approximation is made, which consists in ignoring the displacement current when compared with the conduction current. Also, the magnetic Reynolds number $\mathrm{Rm}=\mu \sigma r_{o} \Omega h$ is considered to be negligibly small, where $\mu$ and $\sigma$ are the magnetic permeability and the electrical conductivity of the liquid metal, respectively (indicative values: $\mu=4 \pi \times 10^{-7} \mathrm{H} . \mathrm{m}^{-1}$, $\sigma=2.3 \times 10^{6}{\mathrm{~S} . \mathrm{m}^{-1}}$, see Liu et al. [2]). This allows us to form the classical weakly coupled MHD model, which results in the fact that the electromotive current is approximated as $\vec{v} \times \vec{B} \sim \vec{v} \times \vec{B}_{0}$, where $\vec{v}$ is the flow velocity, and that the Lorentz force is approximated as $\vec{j} \times \vec{B} \sim \vec{j} \times \vec{B}_{0}$, where $\vec{j}$ is the electric current density. 
When normalizing the MHD equations, the Hartmann number, $\mathrm{Ha}=B_{0} h \sqrt{\sigma / \eta}$, is highlighted.

Finally, several assumptions are made to describe the behavior of the liquid/gas interface. First, the interface is considered to be flat, with a capillary length $l_{c}=\sqrt{\gamma / \rho g} \ll r_{o}-r_{i}$, where $\mathrm{g}$ is the gravity and $\gamma$ is the surface tension of Galinstan (indicative value: $\gamma=0.534 \mathrm{~N} \cdot \mathrm{m}^{-1}$, see Liu et al. [2]). Furthermore, the flow of the liquid surface is modeled through a surface momentum conservation (jump momentum balance: JMB), in which use is made of two rheological parameters: surface shear viscosity, $\eta_{S}$, and surface dilatational viscosity, $\kappa_{S}-$ see Equations (12) and (13). In the case of liquid metals, it is assumed that these parameters only depend on the $\mathrm{O}_{2}$-rate of the surrounding atmosphere (that directly rules interface oxidation), which implicitly requires thermodynamic equilibrium. The hypothesis of a uniform layer of oxidation is put forward which yields that no radial dependence of $\eta_{S}$ or $\kappa_{S}$ is taken into account in this paper.

Due to centrifugal effects, a swirling flow is generated. A distinction is made between the (main) azimuthal flow $v_{\theta}$, and the (secondary) meridian flow $\left(v_{r}, v_{z}\right)$ (also referred to as overturning flow in this paper). When the electroconductive liquid is subjected to an outer magnetic field, electric current densities are induced. Once again, the (main) components $\left(j_{r}, j_{z}\right)$ (due to the main azimuthal flow) are distinguished from the (secondary) component $j_{\theta}$ (due to the secondary meridian flow). The investigation of the interaction between the main and secondary MHD flows is worthwhile, because, depending on the relative value of $\mathrm{Ha}$ and $\mathrm{Re}$, the flow switches from 2-D to 3-D topology. This also greatly affects the surface dynamics, because if $\mathrm{Ha} \gg \mathrm{Re}$, the meridian flow vanishes, and only the azimuthal component of JMB is involved. Consequently, the only relevant rheological parameter is surface shear viscosity $\eta_{S}$, the value of which may dramatically affect the main MHD flow [17]. On the contrary, if the inertial effects are significant, the radial component of JMB is brought into play. Thus, the interface tends to stretch, and the surface dilatational viscosity $\kappa_{S}$ can also affect the surface dynamics. Surface rheology may then modify in turn both the main and the secondary MHD flows.

\subsection{Geometry, governing equations, boundary conditions}

\subsubsection{Geometry}

The geometry of the numerical problem, along with the conditions implemented at the boundaries of the calculation domains, are given in Figure 2.

\subsubsection{Bulk flow}

\section{Electromagnetism}

A potential formulation is used to describe the electromagnetic part of the problem. The basic equations derive from Maxwell's equations, and in particular Ampère's law and the continuity equation, completed by Ohm's law generalized to moving matter. Using now the definition of the electric potential $\phi$ and the magnetic vector potential $\vec{A}$, with respect to the electric field $\vec{E}$ and magnetic induction $\vec{B}$, at steady state:

$$
\vec{E}=-\overrightarrow{\operatorname{grad}}(\phi), \vec{B}=\overrightarrow{\operatorname{curl}}(\vec{A})
$$

Ampère's law can be rewritten as:

$$
\overrightarrow{\operatorname{curl}}(\overrightarrow{\operatorname{curl}}(\vec{A}))-\mu \sigma \vec{v} \times(\overrightarrow{\operatorname{curl}}(\vec{A}))+\mu \sigma \overrightarrow{\operatorname{grad}}(\phi)=0,
$$

and the continuity equation as:

$$
\operatorname{div}(\mu \sigma \vec{v} \times(\overrightarrow{\operatorname{curl}}(\vec{A}))-\mu \sigma \overrightarrow{\operatorname{grad}}(\phi))=0,
$$

completed by the Coulomb gauge to define uniquely $\vec{A}$, i.e. $\operatorname{div}(\vec{A})=0$. Equations (2) and (3) are the solved electromagnetism equations.

The electromagnetic boundary conditions first consist of an externally applied constant axial magnetic field, imposed through an "infinite" box surrounding the fluid area: $\vec{B}_{\infty}=B_{0} \overrightarrow{e_{z}}$. The assumption $\mathrm{Rm} \ll 1$ yields: $B_{z} \overrightarrow{e_{z}} \sim B_{0} \overrightarrow{e_{z}}$, throughout the entire computational domain. The result is, in terms of the magnetic vector potential:

$$
\frac{1}{r} \frac{\partial r A_{\theta}}{\partial r}=B_{0}
$$

The second electromagnetic boundary condition is the electrical insulation at the liquid metal boundaries (side-walls, rotating floor, and liquid/gas interface): $\left.\vec{j} \cdot \vec{n}\right|_{\text {fluid walls }}=0$. Using Ohm's law and the low $R m$ assumption, this condition is written with respect to electric potential:

$$
\left.\sigma\left(-\overrightarrow{\operatorname{grad}}(\phi)+\vec{v} \times \vec{B}_{0}\right)\right|_{\text {fluid walls }} \cdot \vec{n}=0
$$

where $\vec{n}$ is the unit normal vector at the considered boundary - see Figure 2.

\section{Fluid mechanics}

A primitive pressure-velocity formulation is used, based on the complete set of Navier-Stokes equations:

$$
\begin{aligned}
\operatorname{div}(\vec{v}) & =0 \\
\rho(\vec{v} \cdot \overrightarrow{\operatorname{grad}}) \vec{v} & =\operatorname{div}\left(-p \overline{\bar{I}}+\eta\left(\overline{\overline{\operatorname{grad}}}(\vec{v})+\overline{\overline{\operatorname{grad}}}^{\top}(\vec{v})\right)\right)+\vec{F} .
\end{aligned}
$$

The coupling term $\vec{F}$ is the Lorentz force, defined as $\vec{F}=$ $\vec{j} \times \vec{B}$, which can be explicitly written as follows, using Ohm's law and the approximation $\mathrm{Rm} \ll 1: \vec{F}=\vec{j} \times \vec{B}_{0}$. 


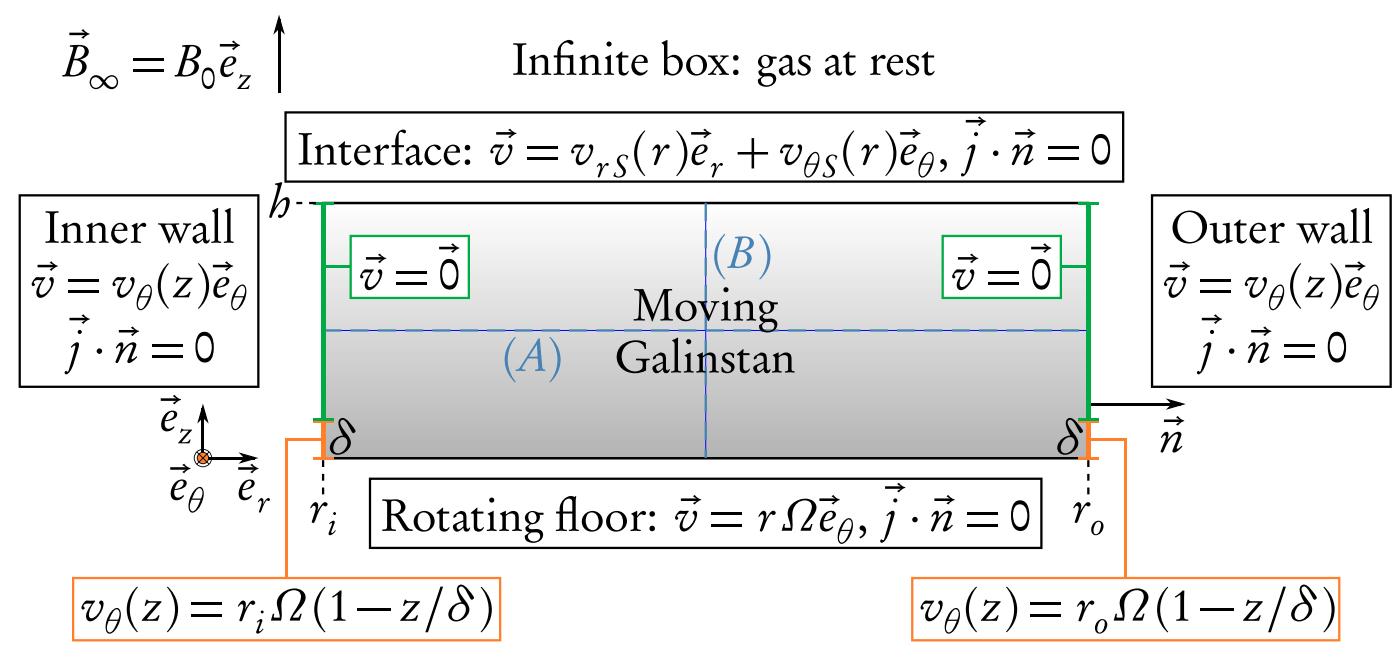

Fig. 2. Geometry and boundary conditions of the channel cross-section used for numerical computation. Note the presence of cutting lines $(A): z=h_{0} / 2$ and $(B): r=\left(r_{i}+r_{o}\right) / 2$, used hereafter for interpretation of the results.

Let us now examine the hydrodynamic boundary conditions, starting with the rotating floor at the bottom of the annular channel:

$$
\vec{v}(r, z=0)=v_{\theta}(r, z=0) \vec{e}_{\theta}=r \Omega \vec{e}_{\theta} \text { for } r \in\left[r_{i}, r_{o}\right] .
$$

At the inner and outer side-walls, a no-slip boundary condition is normally imposed for velocity. However, in order to circumvent the boundary discontinuity between the side-walls and the rotating floor, two matching functions are introduced for the azimuthal component of velocity (see Fig. 2). These functions apply along segments of typical length $\delta \ll r_{i}, r_{o}$ (indicative value: $\delta=2.5 \times 10^{-4} \mathrm{~m}$ ) at the inner and outer walls of the annular channel cross-section. With these matching functions, a Couettelike profile is classically assumed at the matching segments [19], which leads to:

$$
v_{\theta}\left(r=r_{i}, z\right)= \begin{cases}r_{i} \Omega\left(1-\frac{z}{\delta}\right) & \text { if } z \in[0, \delta], \\ 0 & \text { if } z \in[\delta, h],\end{cases}
$$

for the inner wall and to

$$
v_{\theta}\left(r=r_{o}, z\right)= \begin{cases}r_{o} \Omega\left(1-\frac{z}{\delta}\right) & \text { if } z \in[0, \delta], \\ 0 & \text { if } z \in[\delta, h],\end{cases}
$$

for the outer wall. Thus, velocity is imposed such that lubrication conditions along the gaps are taken into account, the continuity of $v_{\theta}$ being warranted at the matching points $z=\delta$ at both walls. These lubrication conditions are not simply a numerical technique. They stem from a true experimental layout, where this gap concretely exists between a rotating dish (rotating floor) and an inert cover (side walls), as described in Figure 3.

The last remaining velocity boundary conditions at the liquid gas/interface are given by the surface rheology equations, governing surface dynamics - see Equations (12) and (13):

$$
\vec{v}(r, z=h)=\vec{v}_{S}(r)=v_{r S}(r) \vec{e}_{r}+v_{\theta S}(r) \vec{e}_{\theta} .
$$

\section{Dimensionless quantities}

As mentioned above, when normalizing MHD equations, two scaling parameters emerge, i.e. $\mathrm{Ha}=B_{0} h \sqrt{\sigma / \eta}$ and $\operatorname{Re}=\rho \Omega r_{o}^{2} / \eta$. A third one can be used instead of Re: the Stuart Number or interaction parameter: $\mathrm{N}=$ $\mathrm{Ha}^{2} /$ Re. Classically in MHD, a (Ha, N) formulation is favored, where $\mathrm{N}$ governs the actual competition between inertial and electromagnetic effects. However, in this paper, Ha and Re are preferred for the following reasons. First, some results are displayed for a purely hydrodynamic flow, meaning that $\mathrm{N}=0$, regardless of the value of the Reynolds number. Moreover, the Couette-like layout of the problem, with an imposed velocity at the rotating floor, makes us tend towards the (Ha, Re) description, since the boundary condition at the bottom of the channel is directly linked to Re through the angular speed $\Omega$. Consequently, by monitoring the Reynolds number, different dynamic configurations are described.

The bulk dimensionless quantities of interest, superscripted ${ }^{\star}$, are then defined as follows:

- the radial coordinate $r^{\star}=\left(r-r_{i}\right) /\left(r_{o}-r_{i}\right)$;

- the axial coordinate $z^{\star}=z / h$;

- the velocity $\vec{v}^{\star}=\vec{v} / \bar{V}$, where $\bar{V}=r_{o} \Omega$. The azimuthal component $v_{\theta}^{\star}$, and the meridian components $\left(v_{r}^{\star}, v_{z}^{\star}\right)$ allow for analysis of the main and secondary MHD bulk flows, respectively;

- the electric current densities: $\vec{j}^{\star}=\vec{j} / \bar{J}$, where $\bar{J}=$ $\sigma B_{0} \bar{V}$. The meridian components $\left(j_{r}^{\star}, j_{z}^{\star}\right)$, and the azimuthal component $j_{\theta}^{\star}$ allow for analysis of the main and secondary MHD bulk flows, respectively;

- and finally, the Lorentz force: $\vec{F}^{\star}=\vec{F} / \vec{F}$, where $\bar{F}=\sigma B_{0}^{2} \bar{V}$. The radial component $F_{\theta}^{\star}$, and the azimuthal component $F_{r}^{\star}$ allow for analysis of the main and secondary MHD bulk flows, respectively. 


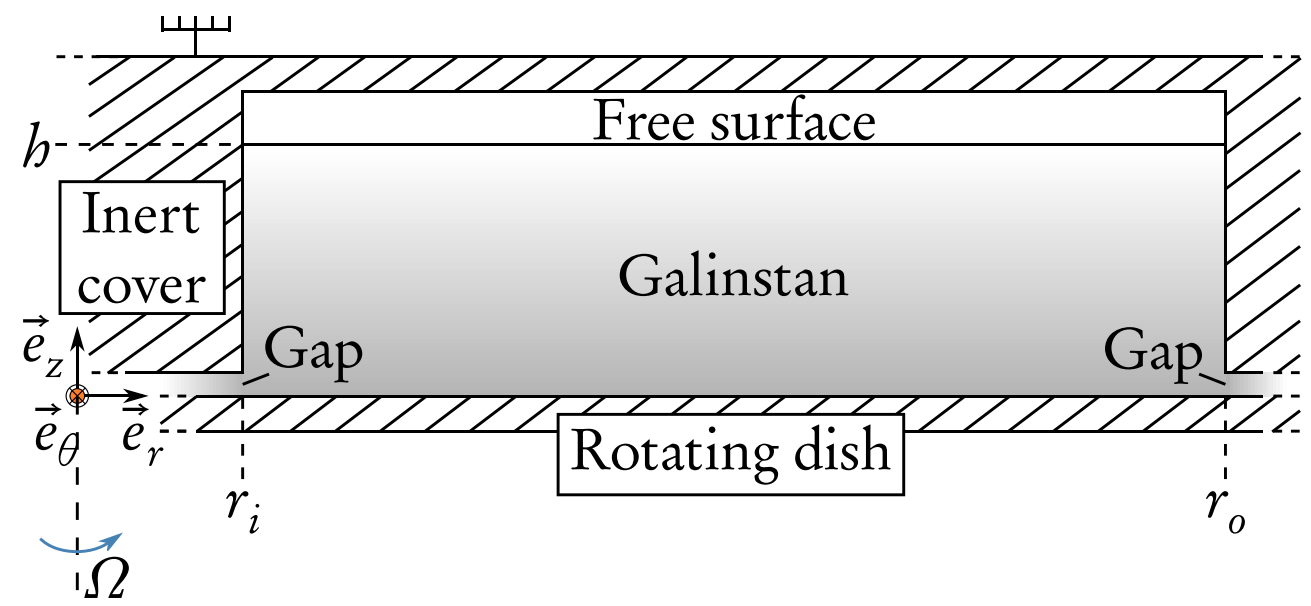

Fig. 3. Lubrication gaps between the rotating floor and the motionless side-walls, with respect to the experimental layout (see e.g. Mannheimer et al. [12]).

\subsubsection{Surface flow}

\section{Surface rheology}

The boundary condition (11) brings a new unknown into play, which is the surface velocity $v_{\theta S}$. This stands as a first coupling term of the two-way coupling between the surface and MHD bulk flow equations. To model the relationship between surface stress and surface strain, the Boussinesq-Scriven constitutive law is used (Aris [20]) to write the two following components of the jump momentum balance (JMB):

$$
\begin{aligned}
\left(\eta_{S}+\kappa_{S}\right)\left(\frac{\mathrm{d}^{2} v_{r S}}{\mathrm{~d} r^{2}}+\frac{1}{r} \frac{\mathrm{d} v_{r S}}{\mathrm{~d} r}-\frac{v_{r S}}{r^{2}}\right) & =\left.\eta \frac{\partial v_{r}}{\partial z}\right|_{z=h}, \\
\eta_{S}\left(\frac{\mathrm{d}^{2} v_{\theta S}}{\mathrm{~d} r^{2}}+\frac{1}{r} \frac{\mathrm{d} v_{\theta S}}{\mathrm{~d} r}-\frac{v_{\theta S}}{r^{2}}\right) & =\left.\eta \frac{\partial v_{\theta}}{\partial z}\right|_{z=h}
\end{aligned}
$$

where $\eta_{S}$ is the surface (in-excess) shear viscosity, and $\kappa_{S}$ is the surface (in-excess) dilatation viscosity. The lefthand term of Equation (12) represents a joint influence of viscous shear and viscous dilatation along the liquid surface, while only the surface viscous shear is present in Equation (13). The right-hand terms are the liquid shears vertically imposed from the supporting sub-phase, with $\eta$, the Newtonian bulk shear viscosity. They stand as the second term of the latter two-way coupling. Equations (12) and (13) are solved along the interface by calculating their respective weak forms, with two Dirichlet end-point boundary conditions:

$$
\begin{aligned}
v_{r S}\left(r=r_{i}\right)=v_{r S}\left(r=r_{o}\right)= & v_{\theta S}\left(r=r_{i}\right) \\
& =v_{\theta S}\left(r=r_{o}\right)=0 .
\end{aligned}
$$

\section{Scaling parameters}

Normalizing Equations (12) and (13) leads to the definition of two rheological scaling parameters:

$$
\mathrm{Bo}_{\eta_{S}}=\frac{\eta_{S}}{\eta h}, \mathrm{Bo}_{\kappa_{S}}=\frac{\kappa_{S}}{\eta h},
$$

where $\mathrm{Bo}_{\eta_{S}}$ and $\mathrm{Bo}_{\kappa_{S}}$ are the surface shear and surface dilatational Boussinesq numbers, respectively. The $\mathrm{Bo}_{\eta} S$ number describes the balance between bulk and surface viscous shears, while $\mathrm{Bo}_{\kappa_{S}}$ expresses the ratio between the dilatational stress along the interface and the bulk viscous shear. It should be noted that, compared to the supporting article [17], $\mathrm{Bo}_{\kappa_{S}}$ is a new feature emerging due to the centrifugal effects.

\subsubsection{Numerical implementation}

With respect to numerical methods, due to the basic layout of the computational domain, a fully-coupled approach is implemented, based on the full Jacobian matrix as one entity. This approach is based on the NewtonRaphson method, which linearizes the non-linear problem based on the current solution, at each iteration. A linear stationary direct solver is implemented to solve the linearized problem, i.e. the MUltifrontal Massively Parallel sparse direct Solver (MUMPS), based on LU factorization (see MUMPS support [21] for further details).

Finally, the implemented mesh is displayed in Figure $4 \mathrm{a}$. It consists of 27524 elements, mainly triangular, with mesh refinement on the Galinstan domain. As shown in Figure 4b, a specific rectangular boundary layer mesh is set up at the boundaries of the fluid domain. Typically, the relative thickness of this layer is set so as to be much lower than the reciprocal of the Hartmann and Reynolds numbers, which both monitor the thickness of the physical boundary layers.

\section{Results and interpretation}

Before any extrapolation to unknown areas, numerical modelling is first systematically benchmarked with asymptotic cases. For the classical annular viscometer layout (i.e. with no applied magnetic field), the first analytical benchmark takes surface viscous shearing into account, in the case of a supporting creeping 


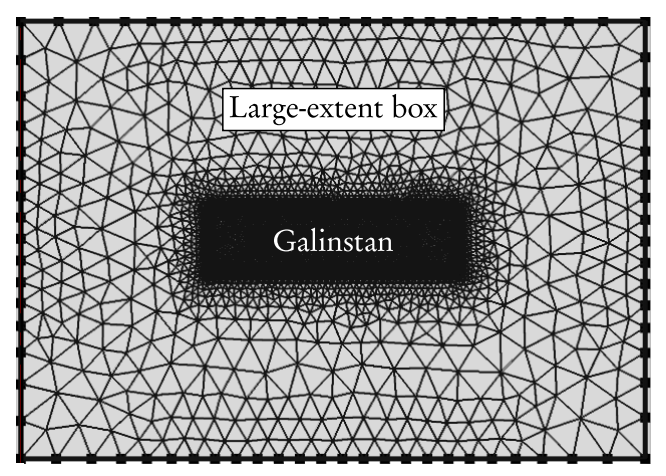

(a)

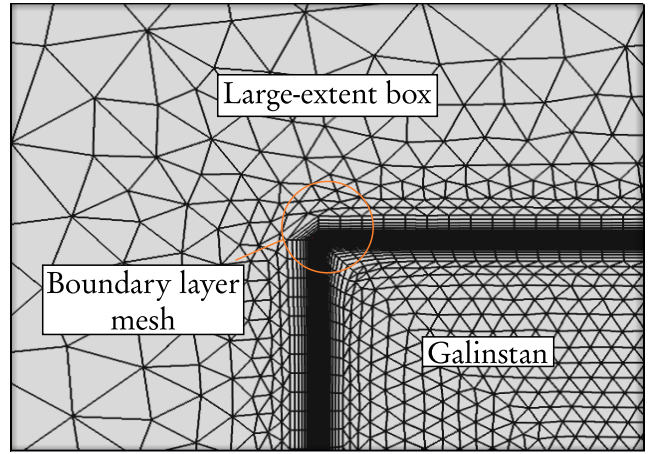

(b)

Fig. 4. Mesh used for the numerical computation: (a) global view, (b) zoom on the boundary layer mesh.

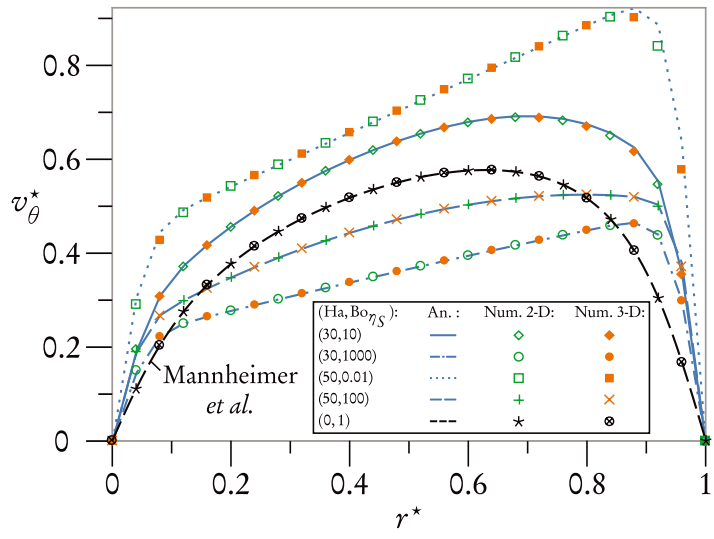

Azimuthal velocity along cutting line $(A)$ (Core \& Shercliff layers), inertia neglected.

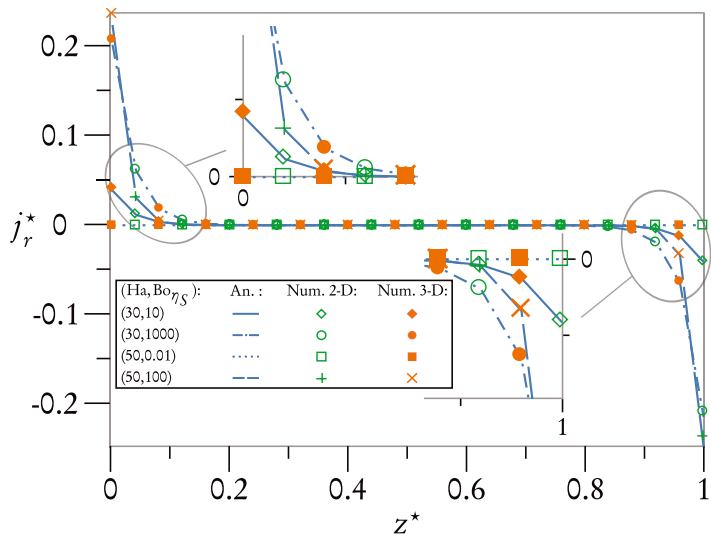

Radial electric current along cutting line $(B)$ (Core \& Hartmann layers), inertia neglected.

Fig. 5. Analytical and numerical results for the annular MHD viscometer, either for purely hydrodynamic creeping flow $($ Ha $=0$ : (a), curves $---[12], *, \otimes)$, or for Ha $\gg$ Re (no inertia: other curves, analytical [17] or numerical modelling). The electric current densities are normalized with respect to the maximum electric current $\bar{J}_{\text {max }}$ reached in all cases, i.e. for Ha $=50$.

flow (Mannheimer et al. [12]). Then, the outer magnetic field is added, and the numerical results are benchmarked with the supporting 2-D analytical study [17], which highlights the interactions between surface viscous shearing, electromagnetism, and creeping flow. Note that in addition to the comparison with the present 3-D numerical study, a second 2-D numerical study is added for the sake of multiple benchmarking, within the asymptotic limit $N \gg 1$ (inertial effects neglected). Once these benchmarking cases have been secured, the interactions between the MHD bulk flow (with inertial effects) and surface rheology (including both surface shearing and dilatation) are discussed.

\subsection{Benchmarking cases}

The analytical and numerical results obtained for the two asymptotic cases previously mentioned are compared in Figure 5. The general agreement between all studies is quite satisfying.

Let us focus on the comparison with the benchmarking MHD analytical study. Analytical, 2-D and 3-D numerical modellings all predict radically different topologies for the MHD flows, depending on the relative values of $\mathrm{Ha}$ and $\mathrm{Bo}_{\eta_{S}}$. If $\mathrm{Ha} \gg \mathrm{Bo}_{\eta_{S}}$, a rigid-body motion, expressing the electromagnetic blocking of the flow, first observed by Lehnert [8], is caused by the well-known two-dimensionality tendency of magnetic induction (see Fig. 5a), case $\left.\left(\mathrm{Ha}, \mathrm{Bo}_{\eta_{S}}\right)=(50,0.01)\right)$. Consequently, the interface is perfectly aligned with the bulk, and the bulk viscous shear at the interface is no longer significant. Therefore, the electric current density is essentially confined to the Shercliff layers, with two electric loops closing up near the side-walls [17].

Now, when $\mathrm{Ha} \ll \mathrm{Bo}_{\eta_{S}}$, the three modelings lead to a quite homogeneous "motionless" configuration (Fig. 5a), case $\left.\left(\mathrm{Ha}, \mathrm{Bo}_{\eta_{S}}\right)=(30,1000)\right)$. This singular phenomenon is partially explained by the fact that, in this case, the surface dynamics is governed by surface viscous shear, and behaves as a non-sliding membrane. Thus, $v_{\theta}^{\star}$ must match with the vanishing component $v_{\theta S}^{\star}$ along the surface. However, this cannot solely account for the motionless layout across the whole cross-section. Other reasons are found by focusing on the electric current densities. 


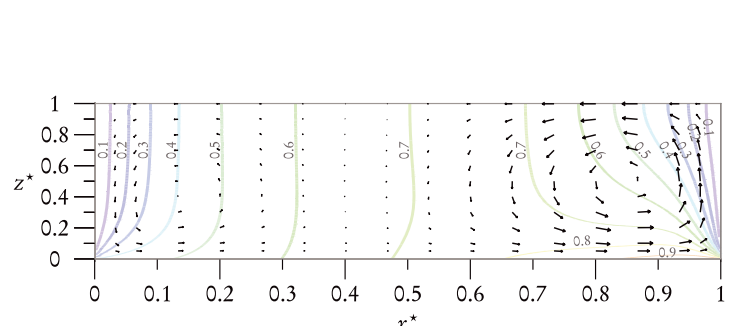

Main (contours: $v_{\theta}^{\star}$ ) and secondary (arrows: $\left.\left(v_{r}^{\star}, v_{z}^{\star}\right)\right)$ flows, $\mathrm{Re}=10^{3}, \mathrm{Ha}=5, \mathrm{Bo}_{\eta_{\mathrm{S}}}=10^{-4}$.

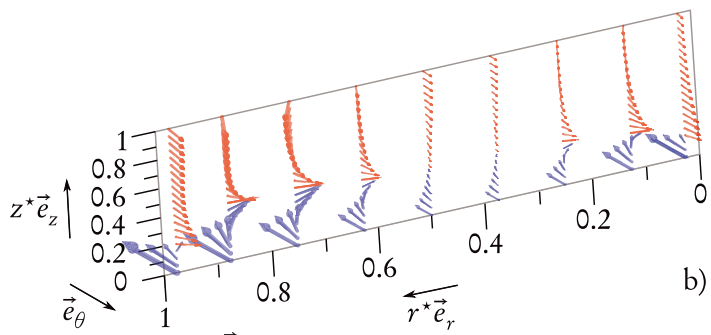

a)

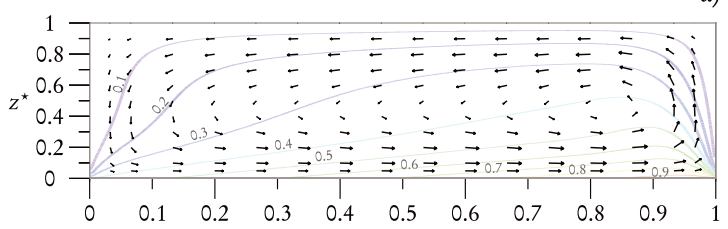
$r^{\star}$

Main (contours: $v_{\theta}^{\star}$ ) and secondary (arrows: $\left.\left(v_{r}^{\star}, v_{z}^{\star}\right)\right)$ flows, $\mathrm{Re}=10^{3}, \mathrm{Ha}=5, \mathrm{Bo}_{\eta_{s}}=10^{4}$.

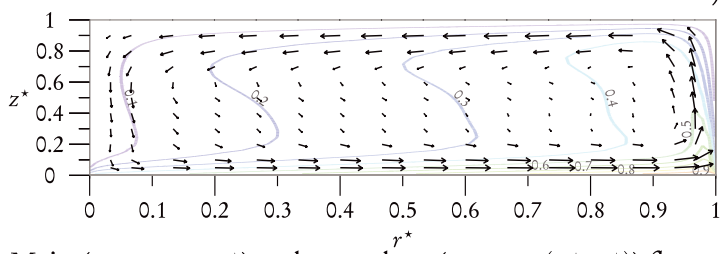

Main (contours: $v_{\theta}^{\star}$ ) and secondary (arrows: $\left.\left(v_{r}^{\star}, v_{z}^{\star}\right)\right)$ flows, $\operatorname{Re}=10^{4}, \mathrm{Ha}=5, \mathrm{Bo}_{\eta_{s}}=10^{4}$.

$\vec{F}^{\star},\left(\mathrm{Re}=10^{3}, \mathrm{Ha}=5, \mathrm{Bo}_{\eta_{s}}=10^{-4}\right)$.

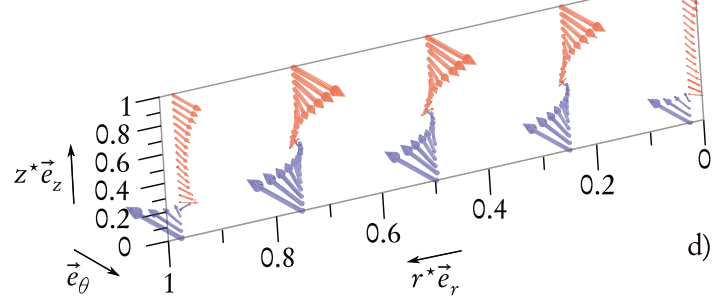

c)

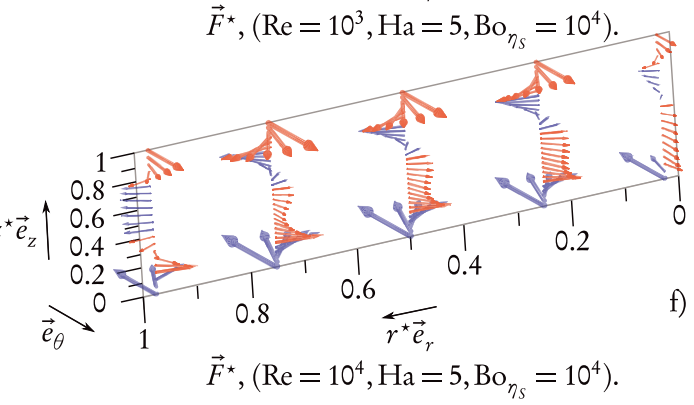

e)

Fig. 6. $\mathrm{Bo}_{\eta_{S}}$ impact on the velocity field $\vec{v}^{\star}$ (left part) and on the Lorentz force $\vec{F}^{\star}$ (right part). ( $\left.v_{r}^{\star}, v_{z}^{\star}\right)$ is log-scaled by the magnitude $\exp \left(\left(\ln \left(\left\|\left(v_{r}^{\star}, v_{z}^{\star}\right)\right\| /\left\|\left(v_{r}^{\star}, v_{z}^{\star}\right)\right\|_{\max }\right)\right) /(1+p)\right) ; p=0.5$ for (a), (c), and (e). $\vec{F}^{\star}$ is log-scaled by the magnitude $\exp \left(\left(\ln \left(\left\|\vec{F}^{\star}\right\| /\left\|\vec{F}^{\star}\right\|_{\max }\right)\right) /(1+p)\right) ; p=1.5$ for (b), (d), and (f). For $\vec{F}^{\star}$, green arrows (when present) are essentially meridian, while red and blue arrows correspond to significantly (i.e. $\left|F_{\theta}^{\star}\right| /\left\|\vec{F}^{\star}\right\| \geq 0.01$ ) positive and negative azimuthal components, respectively.

Due to strong velocity gradients near the liquid/gas surface, and to current continuity, electric current densities are now found to flow within the top and bottom Hartmann layers, which are therefore electrically active - see Figure $5 \mathrm{~b}$. The presence of a strong radial component of electric current density, combined with the imposed magnetic field $B_{0} \vec{e}_{z}$, leads to the emergence of a Lorentz force $-j_{r} B_{0}$ along the azimuthal direction. This Lorentz force is negative at the bottom, and positive at the top of the channel cross-section. Consequently, this leads to an electromagnetic damping of the momentum injected from the rotating floor at the bottom, while it enhances momentum in the top part of the channel. Both contributions lead to a homogenization of the flow, which accounts for the overall flow patterns.

To conclude, we confirm that for the asymptotic case $\mathrm{Ha} \gg \mathrm{Re}$, the surface shear Boussinesq number drives electrical activation of the Hartmann layers. Thus, surface rheology can really monitor the magnitude of both velocity and electric currents in the MHD core and the boundary layers, highlighting the competitive effects between MHD tendency towards two-dimensionality, and surface viscous shearing.

\subsection{Inertia, MHD and surface rheology}

3-D numerical modeling, the reliability of which is demonstrated from the previous asymptotic case, can now be confidently extrapolated to conditions including MHD of an annular swirling flow, coupled with surface rheology.

\subsubsection{Surface viscous shear}

In this section, the influence of surface viscous shearing only (through $\mathrm{Bo}_{\eta_{S}}$ ) on main and secondary MHD bulk flows is investigated, in order to complete the analytical analysis [17]. For this purpose, the surface dilatation Boussinesq number is set to be negligibly small: $\mathrm{Bo}_{\kappa_{S}}=10^{-4}$. In order to analyse the surface viscous shear impact, the velocity field $\vec{v}$ and the Lorentz Force $\vec{F}$ with respect to several ( $\left.\mathrm{Re}, \mathrm{Ha}, \mathrm{Bo}_{\eta_{S}}\right)$ values are displayed in Figure 6.

Concerning the main flow, at a given relatively low Re value, the qualitative effects of surface viscous shearing on the azimuthal velocity and Lorentz force profiles are essentially the same as in the previously detailed benchmarking asymptotic case. For instance, when $\operatorname{Re}=10^{3}$ 
and $\mathrm{Ha}=5$, if $\mathrm{Bo}_{\eta_{S}} \ll \mathrm{Ha}$ (Figs. 6a and 6b), then the fluid flow tends towards a rigid body motion (though not really pronounced as $\mathrm{Ha}$ is not high enough) where the electric current densities are confined within the (thick) Shercliff layers. If $\mathrm{Bo}_{\eta_{S}} \gg \mathrm{Ha}$ (Figs. 6c and 6d), an electromagnetic damping of the flow is also observed (again not so marked), and the Hartmann layers become electrically active, with a (damping) negative Lorentz force at the bottom, and a (driving) positive Lorentz force at the top. Finally, when the Reynolds number is increased (for instance $\mathrm{Re}=10^{4}$, Figs. 6e and $6 \mathrm{f}$ ), the azimuthal velocity profile evolution with respect to $\mathrm{Bo}_{\eta_{S}}$ shows an efficient advection of the main flow by the secondary flow. Increasing Ha does not provide any interesting new features.

Now, as far as the secondary flow is concerned, it is clear from Figures $6 \mathrm{a}$ and $6 \mathrm{c}$ that increasing $\mathrm{Bo}_{\eta_{S}}$ leads to flow homogenization for $\left(v_{r}^{\star}, v_{z}^{\star}\right)$. When $\mathrm{Bo}_{\eta_{S}}=10^{-4}$, i.e. when liquid surface acts similarly to a free surface, the main vortex governing the overturning flow is mainly located in the outer part of the channel. When $\mathrm{Bo}_{\eta_{S}}=$ $10^{4}$, this vortex expands radially inwards throughout the whole cross-section of the channel. This difference can be accounted for by the nature of the boundary condition at the surface. When the latter is similar to a free surface, the momentum injected from the rotating floor is dissipated in the bulk and along the sliding interface, with a significant surface radial velocity. When the liquid surface is rigid (vanishing surface velocity), it no longer participates in viscous damping of the injected momentum. Bulk viscous damping is therefore enhanced, leading to expansion of the main vortex inside the sub-phase. Note that when Re varies at fixed $\left(\mathrm{Ha}, \mathrm{Bo}_{\eta_{S}}\right)$, overturning flow magnitude increases with Re, and the main vortex governing the secondary flow is enlarged. Momentum is then increasingly confined within the Ekman layers (Fig. 6e), resulting into a kind of well-developed helical pattern in the core flow for the Lorentz force (Fig. 6f). This atypical flow pattern is due to a significant Lorentz force radial component, in link with strong centrifugal effects. Finally, note that for given $\left(\mathrm{Re}, \mathrm{Bo}_{\eta_{S}}\right)$ values, an increase in Ha affects both the meridian velocities magnitude and the flow topology. The main vortex governing the meridian flow is increasingly diminished, due to MHD tendency towards two-dimensionality. However, this does not change the qualitative influence of $\mathrm{Bo}_{\eta_{S}}$ on both main and swirling flows.

\subsubsection{Surface viscous dilatation}

Following analysis of the impact of surface viscous shear on the overall MHD bulk flow, we shall now investigate the interactions between surface viscous dilatation and the sub-phase flow. As we focus only on the parameter $\mathrm{Bo}_{\kappa_{S}}$, the surface shear Boussinesq number is set to be negligibly small in this section: $\mathrm{Bo}_{\eta_{S}}=10^{-4}$. In order to analyse the surface viscous dilatation impact, the velocity field $\vec{v}$ and the Lorentz Force $\vec{F}$ with respect to several ( $\left.\mathrm{Re}, \mathrm{Ha}, \mathrm{Bo}_{\kappa_{S}}\right)$ values are displayed in Figure 7.
Figures $6 \mathrm{a}$ and $7 \mathrm{a}$ for velocity (respectively $6 \mathrm{~b}$ and $7 \mathrm{~b}$ for Lorentz force) are compared to analyse surface dilatation impact on the main MHD flow. An increase in $\mathrm{Bo}_{\kappa_{S}}$ does not seem to alter significantly the main MHD flow for these (Re, Ha) values, the azimuthal components of both velocity and Lorentz force remaining quite unchanged. It seems that the main flow is less efficiently advected by the secondary flow.

This observation is confirmed by turning to analysis of the secondary flow for the same (Re, Ha) values. Contrary to what is observed for $\mathrm{Bo}_{\eta_{S}}$, in this case the main vortex governing the secondary flow slightly decreases when $\mathrm{Bo}_{\kappa_{S}}$ increases (comparison between Figs. 6a and 6a). This phenomenon is quite unexpected, because for the secondary flow, the boundary condition at the liquid surface along the radial direction is the same both in the case of an infinite value for $\mathrm{Bo}_{\eta_{S}}$ and for $\mathrm{Bo}_{\kappa_{S}}$, i.e. a vanishing $v_{r S}^{\star}$ (where the interface is similar to a non-sliding wall, see Eq. (12)). However, as $\mathrm{Bo}_{\eta_{S}}$ also governs the azimuthal boundary condition for the main flow, when the surface viscous shear is very high, the main azimuthal flow must also match with a vanishing $v_{\theta S}^{\star}$ (see Eq. (13)). Consequently, deflection of momentum at the interface is complete, favoring expansion of the main overturning vortex as mentioned above (Fig. 6c). For surface dilatation, even when $\mathrm{Bo}_{\kappa_{S}}$ greatly increases, it has no direct consequence on the boundary condition $v_{\theta S}^{\star}$ for the main flow. Therefore, for $\mathrm{Bo}_{\kappa_{S}}=10^{4}$, the overturning flow must match with a vanishing value of $v_{r S}^{\star}$, whereas the main flow remains unchanged at the vicinity of the interface (with a fixed $\mathrm{Bo}_{\eta_{S}}=10^{-4}$ ). This favors slight damping of the main vortex governing the secondary flow near the interface.

Now, when the (Re, Ha) values are increased, some interesting new features appear. Similarly to what is observed for the $\mathrm{Bo}_{\eta_{S}}$ section, when Re increases at fixed $\left(\mathrm{Ha}, \mathrm{Bo}_{\kappa_{S}}\right)$, overturning flow magnitude increases with Re, and the main vortex governing the secondary flow is enlarged (Fig. 7c), favoring main flow advection. The Hartmann layers remaining electrically inactive, the Lorentz force is essentially electromotive, following the variations of $v_{r}^{\star}$ (Fig. $\left.7 \mathrm{~d}\right)$.

However, contrary to the previous section, an increase in Ha really modifies the qualitative influence of $\mathrm{Bo}_{\kappa_{S}}$ on the swirling flow. Concerning the azimuthal velocity, two distinct areas can be distinguished in Figure $7 d$, as summarised in Figure 8. For the inner part of the cross section, where the velocity magnitude is lower, the inertial effects are weak compared to the electromagnetic effects. Consequently, the electromagnetic blocking observed previously in the $2-\mathrm{D}$ benchmarking case predominates. For the outer part, inertia becomes significant, and the advection of $v_{\theta}^{\star}$ by the overturning flow is strong. Concerning the secondary flow, the emergence of new flow patterns is observed, and a counter-rotating vortex appears. The mainly electromotive Lorentz force follows this flow patterns, with a sudden change of sign along the radial direction in the counter-rotating vortex area (Fig. $7 \mathrm{f}$ ). 


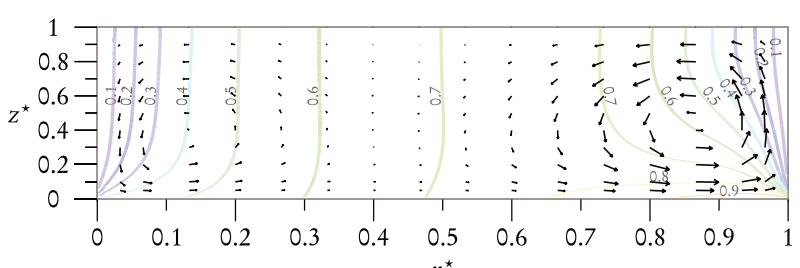

Main (contours: $v_{\theta}^{\star}$ ) and secondary (arrows: $\left.\left(v_{r}^{\star}, v_{z}^{\star}\right)\right)$ flows, $\mathrm{Re}=10^{3}, \mathrm{Ha}=5, \mathrm{Bo}_{x_{S}}=10^{4}$.

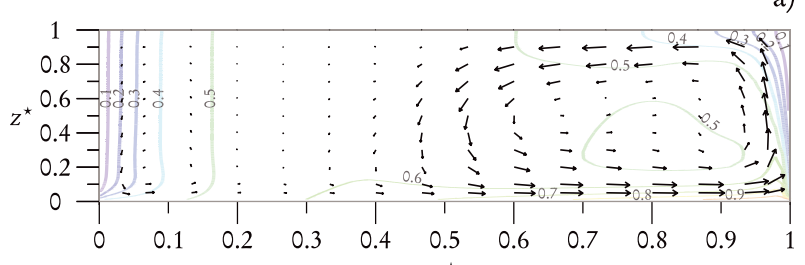

Main (contours: $\left.v_{\theta}^{\star}\right)$ and secondary (arrows: $\left.\left(v_{r}^{\star}, v_{z}^{\star}\right)\right)$ flows, $\mathrm{Re}=10^{4}, \mathrm{Ha}=5, \mathrm{Bo}_{x_{S}}=10^{4}$.

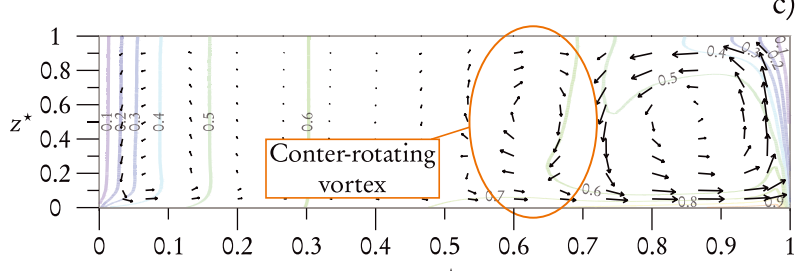

Main (contours: $v_{\theta}^{\star}$ ) and secondary (arrows: $\left.\left(v_{r}^{\star}, v_{z}^{\star}\right)\right)$ flows (circled: emerging contra-rotative vortex)

$$
\mathrm{Re}=10^{4}, \mathrm{Ha}=10, \mathrm{Bo}_{\chi_{S}}=10^{4} .
$$

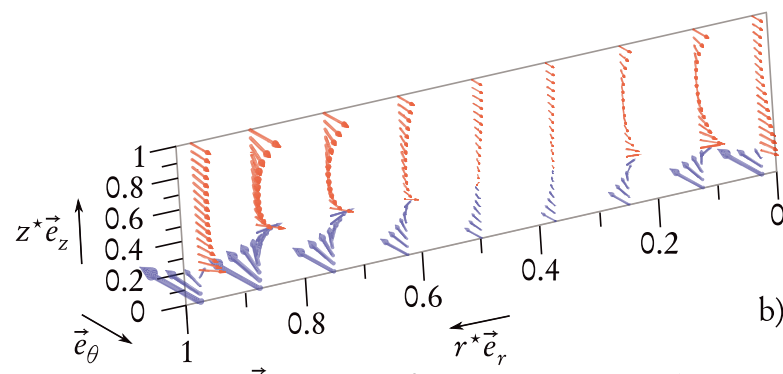

$\vec{F}^{\star},\left(\operatorname{Re}=10^{3}, \mathrm{Ha}=5, \mathrm{Bo}_{\chi_{S}}=10^{4}\right)$.

a)

Fig. 7. $\mathrm{Bo}_{\kappa_{S}}$ impact on the velocity field $\vec{v}^{\star}$ (left part) and on the Lorentz force $\vec{F}^{\star}$ (right part). ( $\left.v_{r}^{\star}, v_{z}^{\star}\right)$ is log-scaled by the magnitude $\exp \left(\left(\ln \left(\left\|\left(v_{r}^{\star}, v_{z}^{\star}\right)\right\| /\left\|\left(v_{r}^{\star}, v_{z}^{\star}\right)\right\|_{\max }\right)\right) /(1+p)\right) ;(\mathrm{a}),(\mathrm{c}): p=0.5,(\mathrm{e}): p=1$. $\vec{F}^{\star}$ is log-scaled by the magnitude $\exp \left(\left(\ln \left(\left\|\vec{F}^{\star}\right\| /\left\|\vec{F}^{\star}\right\|_{\max }\right)\right) /(1+p)\right) ; p=1.5$ for (b), (d), and (f). For $\vec{F}^{\star}$, green arrows (when present) are essentially meridian, while red and blue arrows correspond to significantly (i.e. $\left|F_{\theta}^{\star}\right| /\left\|\vec{F}^{\star}\right\| \geq 0.01$ ) positive and negative azimuthal components, respectively.

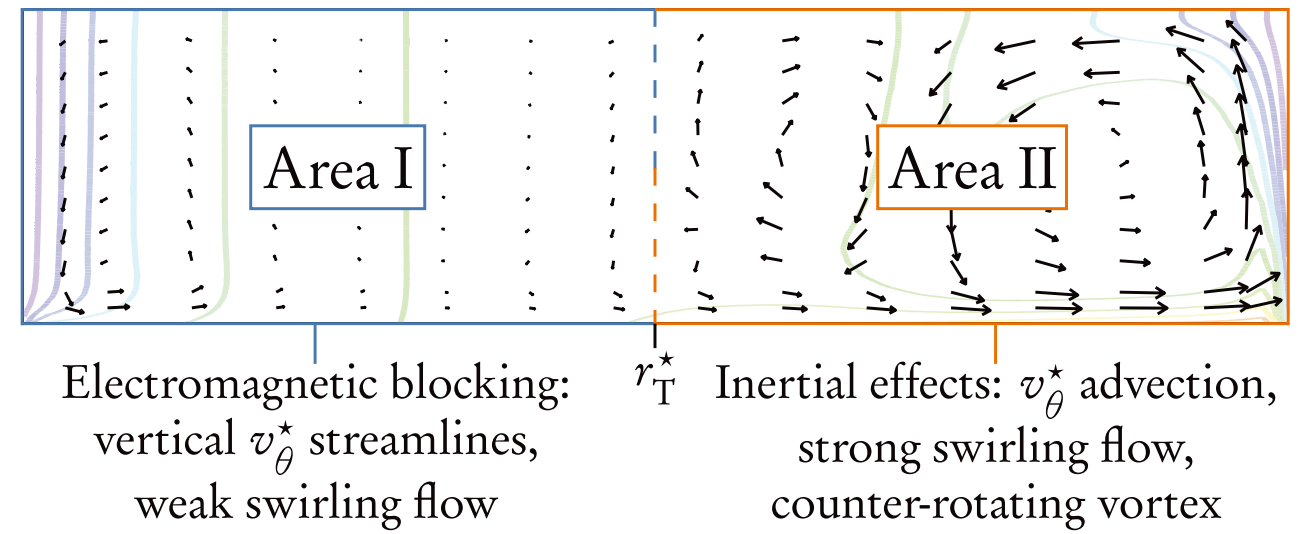

Fig. 8. The two separate flow areas with distinct preponderant physical mechanisms, due to surface viscous dilatation, for $\left(\mathrm{Re}, \mathrm{Ha}, \mathrm{Bo}_{\kappa_{S}}\right)=\left(10^{4}, 5,10^{4}\right) \cdot r_{\mathrm{T}}^{\star}$ corresponds to the transitional radial position. 


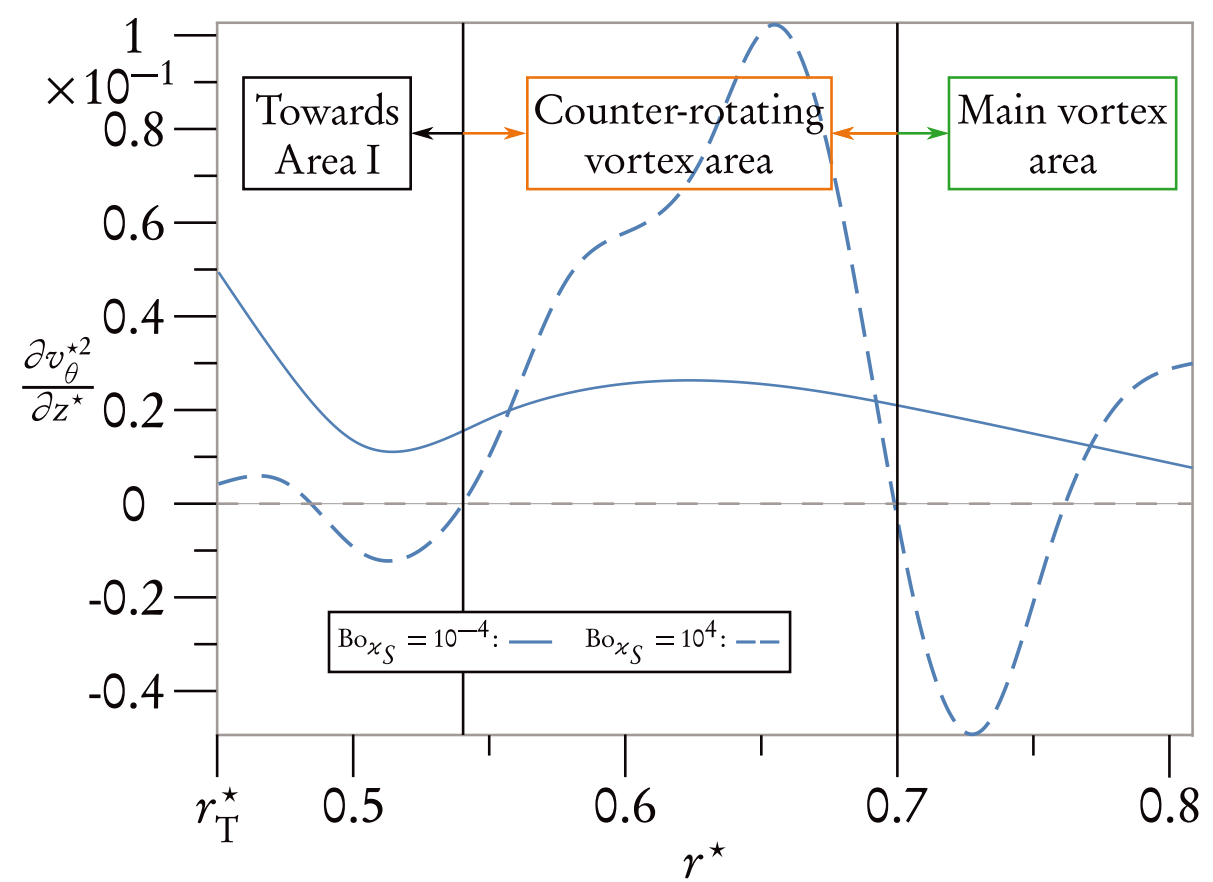

Fig. 9. The curl of the centrifugal force in Area II. $r_{\mathrm{T}}^{\star}$ corresponds to the transitional radial position of Figure 8 .

The emergence of this new vortex seems to stem from a purely hydrodynamic phenomenon. There are several hints endorsing this assumption. First, the radial Lorentz force in this area is found to be always oriented in the opposite direction to the velocity field, due to the Lenz principle. The purely electro-motive radial component of Lorenz force only results in electromagnetic braking of this counter-rotating flow, and as such, cannot generate this new vortex.

Besides, the emergence of this new vortex seems to be directly related to the profile of the curl of the centrifugal force, $\partial v_{\theta}^{\star 2} / \partial z^{\star}$, in area II, as shown in Figure 9. When $\mathrm{Bo}_{\kappa_{S}}=10^{-4}$, this term is everywhere positive, giving rise to the single main vortex ruling the swirling flow. When $\mathrm{Bo}_{\kappa_{S}}=10^{4}$, this term is oscillating, and the changes of sign are strongly linked to the emergence of counterrotating vortices.

Finally, a purely hydrodynamical $(\mathrm{Ha}=0)$ calculation has been performed, by keeping only Area II for the calculation domain. The inner wall boundary condition is modified as such: the velocity profile at $r^{\star}=r_{\mathrm{T}}^{\star}$ resulting from previous MHD calculations is imposed at this fluid boundary. Despite some quantitative differences (due to the lack of Lorentz force for instance), the qualitative aspect of the flow patterns observed in Figure 8 is consistently found in this hydrodynamical calculation.

Consequently, it is the coincidence of the values of the three parameters $\left(\mathrm{Re}, \mathrm{Ha}, \mathrm{Bo}_{\kappa_{S}}\right)$ that leads to this original flow pattern: sufficiently high $\mathrm{Ha}$ and $\mathrm{Bo}_{\kappa_{S}}$ values enhance electromagnetic blocking of the flow in the inner part of the channel (by damping the main vortex governing the secondary flow, thus enhancing the rigid-body motion), whereas a high Re number allows inertial effects to predominate in the outer part of the cross-section. The geometrical configuration of the flow is therefore greatly modified, with the centrifugal flow concentrated against the outer wall, leading to the hydrodynamic development of the latter new counter-rotating vortex.

\section{Conclusion}

To complete the mathematical approach presented in a companion paper [17], which focused only on the interaction between surface viscous shear and a supporting annular MHD flow with no inertia, the overall coupling mechanism between the full viscous rheology of the liquid surface (including surface dilatation) and a supporting annular MHD flow (taking inertial effects into account) is successfully investigated in this paper.

This paper shows how a change in the mechanical properties of a fluid interface can greatly influence a MHD core flow. Moreover, it is proved that shear and dilatational viscosities of the surface do not generate the same changes. On the one hand, viscous shearing of the interface actively modifies the main annular flow by means of the Hartmann layers, which become electrically active. A damping is clearly demonstrated, as is also a 2-D MHD tendency. On the other hand, the dilatational viscosity of the interface is only responsible for damping the overturning flow driven by centrifugation, with a new pattern if inertia and electromagnetic blocking are both significant.

To conclude, the first experimental results obtained so far (that shall be published in a future companion paper) clearly show that the use of a strong magnetic field allows selective measurement of the surface shear viscosity, $\eta_{S}$, thus benchmarking previous results [17]. When the outer field is lowered, inertia competes with electromagnetism. 
The (secondary) centrifugal flow, though, remains very slow at the interface compared to the (main) azimuthal flow, so that satisfying accuracy on radial surface velocity profiles is difficult to achieve. Once these technical limitations (linked to imaging issues) are fixed, the present study shall be used as a benchmarking case to perform selective measurement of surface viscous dilatation, $\kappa_{S}$. As inertial effects strengthen with Re, $\kappa_{S}$ should be more easily determined at relatively high Re, for moderate Ha values.

Acknowledgements. This work was supported by a national $\mathrm{PhD}$ fellow from the French Ministry for Higher Education and Research. The laboratory SIMAP is part of the LabEx Tec 21 (Investissements d'Avenir - Grant agreement No. ANR11-LABX-0030).

\section{References}

[1] J. W. Haverkort, T.W.J. Peeters, Magnetohydrodynamic effects on insulating bubbles and inclusions in the continuous casting of steel, Metall. Mater. Trans. B 41 (2010) $1240-1246$

[2] T. Liu, P. Sen, C.-J. Kim, Characterization of Nontoxic Liquid-Metal Alloy Galinstan for Applications in Microdevices, J. Microelectromech. Syst. 21 (2012) $443-450$

[3] N.B. Morley, S. Smolentsev, L. Barleon, I.R. Kirillov, M. Takahashi, Liquid magnetohydrodynamics: recent progress and future directions for fusion, Fusion Eng. Des. 51-52 (2000) 701-713

[4] V. Kolevzon, G. Gerbeth, Light-scattering spectroscopy of a liquid gallium surface, J. Phys. D 29 (1996) 20712082

[5] V. Kolevzon, G. Gerbeth, G. Podzniakov, Lightscattering study of the mercury liquid-vapor interface, Phys. Rev. E 55 (1997) 3134-3142

[6] R.J. Larsen, M.D. Dickey, G.M. Whitesides, D.A. Weitz, Viscoelastic properties of oxide-coated liquid metals, J. Rheol. 53 (2009) 1305-1326

[7] J.A. Shercliff, Steady motion of conducting fluids in pipes under transverse magnetic field, In Proc. Camb. Phil. Soc., Cambridge University Press, Cambridge, 1953, Vol. 49, pp. 136-144
[8] B. Lehnert, An instability of laminar flow of mercury caused by an external magnetic field, P. Roy. Soc. Lond. A. Mat. 233 (1955) 299-302

[9] R. Moreau, Magnetohydrodynamics, 179-189. Kluwer academic publishers, Dordrecht, 1990, Chaps. 1, 4, 5, pp. $1-31,110-150$

[10] J.C.R. Hunt, K. Stewartson, Magnetohydrodynamic flows in rectangular ducts. II, J. Fluid Mech. 23 (1965) $563-581$

[11] P. Tabeling, J.-P. Chabrerie, Magnetohydrodynamic secondary flows at high Hartmann numbers, J. Fluid Mech. 103 (1981) 225-239

[12] R.J. Mannheimer, R.S. Schechter, An improved apparatus and analysis for surface rheological measurements, J. Colloid Interf. Sci. 32 (1970) 195-211

[13] A.H. Hirsa, J.M. Lopez, R. Miraghaie, Determination of surface shear viscosity via deep-channel flow with inertia, J. Fluid Mech. 470 (2002) 135-149

[14] L. Davoust, Y.-L. Huang, S.-H. Chang, Flow-induced melting of condensed domains within a dispersed Langmuir film, Phys. Fluids 20 (2008) 082105

[15] L. Davoust, Y.-L. Huang, S.-H. Chang, Shearing of a stratified layer of amphiphilic (bio)molecules, Surf. Sci. 603 (2009) 2777-2788

[16] J.M. Lopez, R. Miraghaie, A.H. Hirsa, Non-newtonian behavior of an insoluble monolayer: effects of inertia, J. Colloid Interf. Sci. 248 (2002) 103-110

[17] J. Delacroix, L. Davoust, Electrical activity of the Hartmann layers relative to surface viscous shearing in an annular magnetohydrodynamic flow, Phys. Fluids 26 (2014) 037102-1-037102-21

[18] E. Serre, E. Crespo del Arco, P. Bontoux, Annular and spiral patterns in flows between rotating and stationary discs, J. Fluid Mech. 434 (2001) 65-100

[19] A.J. Pintar, The measurement of surface viscosity, Ph.D. thesis, Illinois Institute of Technology, 1968

[20] R. Aris, Vectors, Tensors and the basic equations of Fluid Mechanics, Prentice-Hall, Englewood Cliffs, NJ, 1962

[21] MUMPS Support. MUltifrontal Massively Parallel Solver (MUMPS 4.10.0) User's guide. Technical report, ENSLyon and ENSEEIHT, 2011 